\title{
The Solvability of Fractional Elliptic Equation with the Hardy Potential
}

\author{
Siyu Gao, ${ }^{1}$ Shuibo Huang $\mathbb{D}{ }^{1,2}$ Qiaoyu Tian $\mathbb{D}^{1},{ }^{1}$ and Zhan-Ping Ma $\mathbb{D}^{3}$ \\ ${ }^{1}$ School of Mathematics and Computer Science, Northwest Minzu University, Lanzhou, Gansu 730030, China \\ ${ }^{2}$ Key Laboratory of Streaming Data Computing Technologies and Application, Northwest Minzu University, Lanzhou, \\ Gansu 730030, China \\ ${ }^{3}$ School of Mathematics and Information Science, Henan Polytechnic University, Jiaozuo, Henan 454003, China \\ Correspondence should be addressed to Shuibo Huang; huangshuibo2008@163.com
}

Received 8 April 2020; Revised 12 May 2020; Accepted 15 May 2020; Published 28 May 2020

Guest Editor: Chun-Lai Li

Copyright (C) 2020 Siyu Gao et al. This is an open access article distributed under the Creative Commons Attribution License, which permits unrestricted use, distribution, and reproduction in any medium, provided the original work is properly cited.

In this paper, we study the existence and nonexistence of solutions to fractional elliptic equations with the Hardy potential $\int(-\Delta)^{s} u-\lambda\left(u /|x|^{2 s}\right)=u^{r-1}+\delta g(u), \quad$ in $\Omega$

$\left\{\begin{array}{ll}u(x)>0, & \text { in } \Omega, \\ u(x)=0, & \text { in } \mathbb{R}^{N} \backslash \Omega,\end{array}\right.$ where $\Omega \subset \mathbb{R}^{N}$ is a bounded Lipschitz domain with $0 \in \Omega,(-\Delta)^{s}$ is a fractional Laplace operator, $s \in(0,1), N>2 s, \delta$ is a positive number, $2<r<r(\lambda, s) \equiv\left(N+2 s-2 \alpha_{\lambda} / N-2 s-2 \alpha_{\lambda}\right)+1, \alpha_{\lambda} \in(0,((N-2 s) / 2))$ is a parameter depending on $\lambda, 0<\lambda<\Lambda_{N, s}$, and $\Lambda_{N, s}=2^{2 s}\left(\Gamma^{2}((N+2 s) / 4)\right) /\left(\Gamma^{2}((N-2 s) / 4)\right)$ is the sharp constant of the Hardy-Sobolev inequality.

\section{Introduction}

In this paper, we consider the solvability of the following fractional elliptic problem:

$$
\begin{cases}(-\Delta)^{s} u-\lambda \frac{u}{|x|^{2 s}}=u^{r-1}+\delta g(u), & \text { in } \Omega, \\ u>0, & \text { in } \Omega, \\ u=0, & \text { in } \mathbb{R}^{N} \backslash \Omega,\end{cases}
$$

where $\Omega \subset \mathbb{R}^{N}$ is a bounded Lipschitz domain with $0 \in \Omega$, $s \in(0,1), \quad N>2 s, \quad 2<r<r(\lambda, s) \equiv\left(\left(N+2 s-2 \alpha_{\lambda}\right) /(N-2 s-\right.$ $\left.\left.2 \alpha_{\lambda}\right)\right)+1, \quad 0<\lambda<\Lambda_{N, s}, \quad$ and $\quad \Lambda_{N, s}=2^{2 s}\left(\left(\Gamma^{2}((N+2 s) /\right.\right.$ $\left.4)) /\left(\Gamma^{2}((N-2 s) / 4)\right)\right)$ is the sharp constant of the Hardy-Sobolev inequality; the fractional Laplace operator $(-\Delta)^{s}$ is defined by

$$
(-\Delta)^{s} u=C(N, s) \text { P.V. } \int_{\mathbb{R}^{N}} \frac{u(x)-u(y)}{|x-y|^{N+2 s}} \mathrm{~d} y,
$$

where P.V. stands for the Cauchy principal value and constant $C(N, s)$ is a constant.

Recently, much attention has been devoted to the study of fractional Laplacian equations. One of the reasons comes from the fact that the fractional Laplacian arises in various areas and different applications, such as phase transitions, finance, stratified materials, flame propagation, ultrarelativistic limits of quantum mechanics, and water waves. For more details, see [1-6] and references therein.

For fractional elliptic problems with the Hardy potential, Abdellaoui et al. [7] obtained the existence and summability of solutions to a class of nonlocal elliptic problem:

$$
\begin{cases}(-\Delta)^{s} u-\lambda \frac{u}{|x|^{2 s}}=f(x, u), & \text { in } \Omega, \\ u>0, & \text { in } \Omega, \\ u=0, & \text { in } \mathbb{R}^{N} \backslash \Omega,\end{cases}
$$


with $f \in L^{m}(\Omega)$ and $0<\lambda<\Lambda_{N, s}$. They mainly considered the summability of solutions to (3) with $f(x, u)=f(x)$ and the existence and regularity of solutions to (3) with $f(x, u)=h(x) / u^{\sigma}$. Mi et al. [8] obtained the combined influence of the Hardy potential and lower order terms on the existence and regularity of solutions to the problem:

$$
\begin{cases}(-\Delta)^{s} u-\lambda \frac{u}{|x|^{2 s}}+u^{p}=f(x), & \text { in } \Omega, \\ u>0, & \text { in } \Omega, \\ u=0, & \text { in } \mathbb{R}^{N} \backslash \Omega .\end{cases}
$$

Barrios et al. [9] discussed the existence and multiplicity of solutions to the following fractional elliptic equation:

$$
\begin{cases}(-\Delta)^{s} u-\lambda \frac{u}{|x|^{2 s}}=u^{p}+\mu u^{q}, & \text { in } \Omega, \\ u>0, & \text { in } \Omega, \\ u=0, & \text { in } \mathbb{R}^{N} \backslash \Omega,\end{cases}
$$

where $0<\lambda<\Lambda_{N, s}, 0<q<1$,

$$
1<p<p(\lambda, s)=\frac{N+2 s-2 \alpha_{\lambda}}{N-2 s-2 \alpha_{\lambda}},
$$

and $\alpha_{\lambda} \in(0,(N-2 s / 2))$ is a parameter depending on $\lambda$. They shown that problem (5) has at least one solution if $1<p<p(\lambda, s)$ and problem (5) has no solution if $p>p(\lambda, s)$.

Recently, Shang et al. [10] studied the existence and multiplicity of positive solutions to the following problem:

$$
(-\Delta)^{s} u-\mu \frac{u}{|x|^{2 s}}=\lambda g(x) u^{p}+K(x) u^{2 *-1}, \quad \text { in } \mathbb{R}^{N},
$$

where $s \in(0,1), N>2 s, 0<p<2_{s}^{*}-1$, and $0<\mu<\Lambda_{N, s}$. Some other results of fractional elliptic equations with the Hardy potential, see $[7,9,11-14]$ and references therein.

The local version of quasilinear problem related to problem (8) has been considered by Boccardo et al. [15]. They analyzed the existence of nontrivial solutions to the following problem:

$$
\begin{cases}-\Delta_{p} u=|u|^{r-2} u+\lambda g(u), & \text { in } \Omega, \\ u>0, & \text { in } \Omega, \\ u=0, & \text { on } \partial \Omega,\end{cases}
$$

where $\Omega \subset \mathbb{R}^{N}$ is a smooth bounded domain, $1<p<N$, $r>p, g: \Omega \times \mathbb{R} \longrightarrow \mathbb{R}$ is a Carathéodory function, and there exist constants $c_{1}>0$ and $q \in(1, p)$ such that $g(s) \leq c_{1} s^{q-1}$ for any $s>0$.

Motivated by the above works, the aim of this paper is to study the existence of solutions to problem (1) by the method of subsuper solutions and taking into advantage the combined effect of concave and convex nonlinearity.

We make the following assumptions:
$(F 1)$

$$
2<r<r(\lambda, s) \equiv \frac{N+2 s-2 \alpha_{\lambda}}{N-2 s-2 \alpha_{\lambda}}+1,
$$

where $\alpha_{\lambda} \in(0,((N-2 s) / 2))$ is a parameter depending on $\lambda$.

(F2) $g: \Omega \times \mathbb{R} \longrightarrow \mathbb{R}$ is Carathéodory function, and there exist constants $c_{1}>0$ and $q \in(1,2)$, such that, for any $\sigma>0$,

$$
g(\sigma) \leq c_{1} \sigma^{q-1}
$$

(F3) The function

$$
u \longrightarrow u^{r-1}+\delta g(u) \text { is nondecreasing. }
$$

(F4) Define

$$
M_{0}=\underline{\lim }_{z \longrightarrow 0^{+}} \frac{g(z)}{z}
$$

for all $\tau>0$,

$$
M_{1}(\tau)=\inf _{0 \leq z \leq\left(\tau \lambda_{1}\right)^{1 /(r-2)}} \frac{g(z)}{z},
$$

where $\lambda_{1}$ is the first eigenvalue of $(-\Delta)^{s}$ in $\Omega$.

Now, we state our main result.

Theorem 1. Suppose $(F 1)-(F 4)$ hold. Then, there exists a positive constant $\delta_{0}$, such that, for all $\delta \in\left(\lambda_{1} / M_{0}, \delta_{0}\right]$, problem (1) has at least a nonnegative solution if $M_{0}>\left(\lambda_{1} / \delta_{0}\right)$, where $M_{0}$ is defined by (12).

Remark 1. In order to prove the above theorem, we study directly to the pseudodifferential operator, without the harmonic extension to an extra dimension by transforming the nonlocal problem into a local problem due to Caffarelli and Silvestre [16].

Remark 2. To establish the upper bound for $r$ (see (9)), we consider a radial solution $w=A|x|^{((2 s-N) / 2)+\beta}$ with constant $A>0$ to the problem:

$$
(-\Delta)^{s} w-\lambda \frac{w}{|x|^{2 s}}=w^{r-1}
$$

We obtain

$$
\begin{gathered}
A \gamma_{\beta}|x|^{-2 s+((2 s-N) / 2)+\beta}-\lambda A|x|^{-2 s+((2 s-N) / 2)+\beta} \\
=A^{r-1}|x|^{(((2 s-N) / 2)+\beta)(r-1)}
\end{gathered}
$$


where

$$
\gamma_{\beta}:=\frac{\pi^{2 s} \Gamma((N+2 s+2 \beta) / 4) \Gamma((N+2 s-2 \beta) / 4)}{\Gamma((N-2 s-2 \beta) / 4) \Gamma((N-2 s+2 \beta) / 4)} .
$$

In order to have homogeneity, we have

$$
\frac{2 s-N}{2}+\beta=\frac{-2 s}{r-2} \text {. }
$$

Thus, we deduce that $\gamma_{\beta}-\lambda=A^{r-2}$. Since $A>0$, we conclude that $\gamma_{\beta}-\lambda>0$. Note that the map $\gamma:[0,((N-$ $2 s) / 2)) \longmapsto\left(0, \Lambda_{N, s}\right]$ is decreasing about $\beta$, see $[17,18]$. Hence, there is a unique element $\alpha_{\lambda}$ such that $\gamma_{\alpha_{\lambda}}=\lambda$. Thus, we have $\alpha_{\lambda}>\beta$, that is,

$$
\alpha_{\lambda}>\frac{-2 s}{r-2}+\frac{N-2 s}{2},
$$

which implies that

$$
r<\frac{N+2 s-2 \alpha_{\lambda}}{N-2 s-2 \alpha_{\lambda}}+1:=r(\lambda, s) .
$$

Therefore, we can construct a supersolution to problem (1) for $r<r(\lambda, s)$, just modifying the $w$ found above. Thus, $r(\lambda, s)$ is the threshold for the existence to problem (1).
Now, we consider the nonexistence of solution to problem (1).

Theorem 2. Suppose (F1) - (F4) hold. Then, problem (1) has no solution in $H_{0}^{s}$ if for some $\tau>1, M_{1}(\tau)>0$, and $\delta>\lambda_{1} / M_{1}(\tau)$, where $M_{0}$ and $M_{1}$ are defined by (12) and (13), respectively.

The following two examples also appeared in [15].

Remark 3. An example of function $g(\sigma) \equiv \sigma^{q}$ with $0<q<1$, which satisfies conditions (10) and (11) for any $\delta \in\left(0, \delta_{0}\right]$, such that problem (1) has at least one positive solution. In this condition $M_{0}=\infty$, by (52), we have $\eta^{r-q} C_{1}^{-1} C_{1}^{2-q}\left(C_{1}-C_{1}^{r}\right) \geq \delta$. Define

$$
\Phi\left(C_{1}\right)=\eta^{r-q} c_{1}^{-1} C_{1}^{2-q}\left(C_{1}-C_{1}^{r}\right) .
$$

It is easy to see that

$$
\frac{d}{d C_{1}} \Phi\left(C_{1}\right)=0 \Longleftrightarrow C_{1}=C_{1,0}:=\left(\frac{3-q}{2-q+r}\right)^{1 /(r-1)} .
$$

We have to prove that $\delta$ is smaller than the minimum of $\Phi\left(C_{1}\right)$. Therefore, we have

$$
\begin{array}{r}
\delta_{0}=\Phi\left(C_{1,0}\right)=\eta^{r-q} c_{1}^{-1}\left(\frac{3-q}{2-q+r}\right)^{1 /(r-1)}\left[\left(\frac{3-q}{2-q+r}\right)^{(2-q) /(r-1)}-\left(\frac{3-q}{2-q+r}\right)^{(1-q+r) /(r-1)}\right] . \\
\delta>\lambda_{1}^{(r-\theta-1) /(r-2)}\left(1+\lambda_{1}^{\alpha /(r-2)}\right) .
\end{array}
$$

Moreover, by (10) and (13), we have for any $\tau>1$,

$$
M_{1}(\tau) \delta_{0} \leq M_{1}(1) \delta_{0} \leq c_{1} \delta_{0} \inf _{0<z \leq \lambda_{1}^{1 /(r-2)}}|z|^{q-2} \equiv c_{1} \delta_{0} \lambda_{1}^{(q-2) /(r-2)}
$$

Thus, for any $\tau>1, M_{1}(\tau) \leq c_{1} \lambda_{1}^{(q-2) /(r-2)}$. Hence, problem (1) has no solution at least $\delta>c_{1} \lambda_{1}^{(r-q) /(r-2)}$. Therefore, the result of the above theorem is more general than [9].

Remark 4. We consider the function

$$
g(\sigma)=\frac{\sigma|\sigma|^{\theta-1}}{1+\sigma|\sigma|^{\alpha-1}}
$$

for $0<\alpha<\theta<1$. We easily deduce that conditions (10) and (11) are fulfilled and $M_{0}=\infty$. On the contrary,

$$
M_{1}(\tau)=\inf _{0 \leq z \leq\left(\tau \lambda_{1}\right)^{1 /(r-2)}} \frac{z^{\theta-1}}{1+z^{\alpha}} .
$$

If $\alpha>\theta-1$, the function $z^{\theta-1} /\left(1+z^{\alpha}\right)$ is monotone decreasing for $z \geq 0$. Then,

$$
M_{1}(\tau)=\frac{\left(\tau \lambda_{1}\right)^{(\theta-1) /(r-2)}}{1+\left(\tau \lambda_{1}\right)^{\alpha /(r-2)}}
$$

Similarly, in this case, problem (1) has no solution provided
Remark 5. The function $\tau \longrightarrow M_{1}(\tau)$ is nonincreasing. Hence, if $\delta>\lambda_{1} / M_{1}\left(\tau_{0}\right)$, for some $\tau_{0}>1$, the results of Theorem 1 will be true for any $\tau>\tau_{0}$.

The paper is organized as follows. In Section 2, we present some definitions and preliminary tools, which will be used in the Proof of Theorems 1 and 2. The Proof of Theorems 1 and 2 are given in Section 3 and Section 4, respectively.

\section{Preliminaries and Function Setting}

In this section, we recall some known results for reader's convenience.

Denote the space

$$
\mathscr{L}^{s}:=\left\{u: \mathbb{R}^{N} \longrightarrow \mathbb{R} \text { is measurable: } \int_{\mathbb{R}^{N}} \frac{|u(x)|}{\left(1+|x|^{N+2 s}\right)} \mathrm{d} x<\infty\right\},
$$

equipped with the norm

$$
\|u\|_{\mathscr{L}^{s}}:=\int_{\mathbb{R}^{N}} \frac{|u(x)|}{\left(1+|x|^{N+2 s}\right)} \mathrm{d} x .
$$


Let $\Omega$ be an open subset of $\mathbb{R}^{N}$. Given $u \in \mathscr{L}^{s}$ and $\varphi$ in the Schwartz class, the distribution $(-\Delta)^{s} u$ in $\mathscr{D}^{\prime}(\Omega)$ is defined as

$$
\left\langle(-\Delta)^{s} u, \varphi\right\rangle=\int_{\mathbb{R}^{N}} u(-\Delta)^{s} \varphi \mathrm{d} x, \quad \text { for any } \varphi \in C_{c}^{\infty}(\Omega) .
$$

We give some useful facts for the fractional Sobolev space.

Definition 1. Let $s \in(0,1)$, and define the fractional Sobolev space:

$$
H^{s}\left(\mathbb{R}^{N}\right)=\left\{u \in L^{2}\left(\mathbb{R}^{N}\right):|\xi|^{s} \widehat{u} \in L^{2}\left(\mathbb{R}^{N}\right)\right\} .
$$
as

We need to consider the space $X_{0}^{s}(\Omega)$, which is defined

$$
X_{0}^{s}(\Omega)=\left\{u \in H^{s}\left(\mathbb{R}^{N}\right), u=0 \text { a.e. in } \mathbb{R}^{N} \backslash \Omega\right\},
$$

with the norm

$$
\|u\|_{X_{0}^{s}(\Omega)}=\left(\int_{Q} \frac{|u(x)-u(y)|^{2}}{|x-y|^{N+2 s}} \mathrm{~d} x \mathrm{~d} y\right)^{1 / 2},
$$

where $Q=\mathbb{R}^{2 N} \backslash\left(\Omega^{c} \times \Omega^{c}\right)$. The pair $\left(X_{0}^{s}(\Omega)\right.$, $\left.\|\cdot\|_{X_{0}^{s}(\Omega)}\right)$ yields a Hilibert space (see Lemma 7 in [19]).

We have to use the classical Sobolev theorem.

Theorem 3 (see [20], Theorem 6.5). Let $s \in(0,1)$, then there exists a positive constant $S=S(N, s)$, such that, for any measurable and compactly supported function $u: \mathbb{R}^{N} \longrightarrow \mathbb{R}$, we have

$$
\|u\|_{L^{2^{*}}\left(\mathbb{R}^{N}\right)}^{2} \leq S \int_{\mathbb{R}^{N}} \int_{\mathbb{R}^{N}} \frac{|u(x)-u(y)|^{2}}{|x-y|^{N+2 s}} \mathrm{~d} x \mathrm{~d} y,
$$

where $2_{s}^{*}$ is the so-called Sobolev critical exponent.

In this paper, we consider the existences of energy solution to problem (1) with the critical and subcritical cases.

Definition 2. We say that $u \in X_{0}^{s}(\Omega)$ is an energy solution to problem (1), if for any $\varphi \in X_{0}^{s}(\Omega)$,

$$
\begin{aligned}
& \int_{\Omega}|u|^{r-2} u \varphi \mathrm{d} x<\infty \\
& \int_{\Omega} \frac{u \varphi}{|x|^{2 s}} \mathrm{~d} x<\infty \\
& \frac{C(N, s)}{2} \int_{Q} \frac{(u(x)-u(y))(\varphi(x)-\varphi(y))}{|x-y|^{N+2 s}} \mathrm{~d} x \mathrm{~d} y \\
& -\lambda \int_{\Omega} \frac{u \varphi}{|x|^{2 s}} \mathrm{~d} x \\
& =\int_{\Omega} u^{r-1} \varphi \mathrm{d} x+\delta \int_{\Omega} g(u) \varphi \mathrm{d} x .
\end{aligned}
$$

We also need to consider the weak solution to problem (1).
Definition 3. We say that $u \in L^{1}(\Omega)$ is a weak solution to problem (1), if $u \geq 0$ a.e. in $\Omega, u=0$ in $\mathbb{R}^{N} \backslash \Omega$,

$$
\int_{\Omega}\left(\lambda \frac{u}{|x|^{2 s}}+u^{r-1}+\delta g(u)\right) \delta^{s} \mathrm{~d} x<\infty,
$$

and for all $\varphi \in C^{2 s+\beta}(\Omega) \cap C^{s}(\bar{\Omega}), \beta>0$,

$$
\int_{\Omega} u(-\Delta)^{s} \varphi \mathrm{d} x=\int_{\Omega}\left(\lambda \frac{u}{|x|^{2 s}}+u^{r-1}+\delta g(u)\right) \varphi \mathrm{d} x,
$$

where $\varphi=0$ in $\mathbb{R}^{N} \backslash \Omega$ and $\delta(x):=\operatorname{dist}(x, \partial \Omega)$.

Definition 4. If $u$ satisfies

$$
\begin{cases}(-\Delta)^{s} u-\lambda \frac{u}{|x|^{2 s}} \geq u^{r-1}+\delta g(u), & \text { in } \Omega, \\ u \geq 0, & \text { in } \mathbb{R}^{N} \backslash \Omega,\end{cases}
$$

in the weak sense, we say that $u$ is a supersolution to problem (1).

If $u$ satisfies

$$
\begin{cases}(-\Delta)^{s} u-\lambda \frac{u}{|x|^{2 s}} \leq u^{r-1}+\delta g(u), & \text { in } \Omega, \\ u \leq 0, & \text { in } \mathbb{R}^{N} \backslash \Omega,\end{cases}
$$

in the weak sense, we say that $u$ is a subsolution to problem (1).

Now, we recall the comparison lemma.

Lemma 1 (see [9]). Let $u \in H^{s}\left(\mathbb{R}^{N}\right)$ and $v \in H^{s}\left(\mathbb{R}^{N}\right)$ be solutions, respectively, to

$$
\begin{aligned}
& \begin{cases}(-\Delta)^{s} u=f_{1}, & \text { in } \Omega, \\
u=g_{1}, & \text { in } \mathbb{R}^{N} \backslash \Omega,\end{cases} \\
& \begin{cases}(-\Delta)^{s} v=f_{2}, & \text { in } \Omega, \\
v=g_{2}, & \text { in } \mathbb{R}^{N} \backslash \Omega .\end{cases}
\end{aligned}
$$

Then, $u(x) \leq v(x)$ for all $x \in \mathbb{R}^{N}$ if $f_{1} \leq f_{2}$ and $g_{1} \leq g_{2}$.

For the supercritical case, we need a prior regularity result, see [9], Lemma 2.2.

Lemma 2. Given $f \in L^{1}\left(\Omega, \delta^{s}(x) d x\right)$, where $\delta(x)=$ $\operatorname{dist}(x, \partial \Omega)$. There exists a unique weak solution $v(x) \in$ $L^{1}(\Omega)$ to

$$
\begin{cases}(-\Delta)^{s} v=f, & \text { in } \Omega, \\ v=0, & \text { in } \mathbb{R}^{N} \backslash \Omega,\end{cases}
$$

in the sense that

$$
\int_{\Omega} v(-\Delta)^{s} \vartheta=\int_{\Omega} f \vartheta,
$$

for all $\vartheta \in C^{2}(\bar{\Omega})$ with $\vartheta=0$ in $\mathbb{R}^{N} \backslash \Omega$.

Moreover, $\|v\|_{L^{1}(\Omega)} \leq C\|f\|_{L^{1}\left(\Omega, \delta^{s}(x) \mathrm{d} x\right)}$, for some constant $C$ independent of $f$. In addition, if $f \geq 0$ 


$$
\begin{cases}(-\Delta)^{s} v=f, & \text { in } \Omega, \\ v \geq 0, & \text { in } \mathbb{R}^{N} \backslash \Omega .\end{cases}
$$

Then, $v \geq 0$ a.e. in $\Omega$.

\section{The Existence Result}

We are now ready to prove Theorem 1 by employing the idea contained in $[9,15]$, whose proof will be split into several steps.

Proof of Theorem 1.

Step 1: subsolution to problem (1). We first consider the eigenvalue problem:

$$
\begin{cases}(-\Delta)^{s} \varphi_{1}=\lambda_{1} \varphi_{1}, & \text { in } \Omega, \\ \varphi_{1}=0, & \text { in } \mathbb{R}^{N} \backslash \Omega .\end{cases}
$$

Note that the eigenfunction $\varphi_{1} \geq 0$ belongs to $X_{0}^{s} \cap L^{\infty}(\Omega)$.

Suppose $\delta M_{0}>\lambda_{1}$, where $M_{0}$ is given in (12), by (F4), for all $\delta \in\left(\lambda_{1} / M_{0}, \delta_{0}\right]$, taking $t$ small enough, we have

$$
\frac{g\left(t \varphi_{1}\right)}{t \varphi_{1}}>\frac{\lambda_{1}}{\delta}
$$

Therefore, for $x \in \Omega$,

$$
(-\Delta)^{s}\left(t \varphi_{1}\right)=\lambda_{1} t \varphi_{1}<\delta g\left(t \varphi_{1}\right) \leq \delta g\left(t \varphi_{1}\right)+\left(t \varphi_{1}\right)^{r-1}+\lambda \frac{t \varphi_{1}}{|x|^{2 s}} .
$$

Therefore,

$$
\begin{cases}(-\Delta)^{s}\left(t \varphi_{1}\right) \leq \delta g\left(t \varphi_{1}\right)+\left(t \varphi_{1}\right)^{r-1}+\lambda \frac{t \varphi_{1}}{|x|^{2 s},} & \text { in } \Omega, \\ t \varphi_{1}=0, & \text { in } \mathbb{R}^{N} \backslash \Omega .\end{cases}
$$

Thus, $\underline{u}:=t \varphi_{1}$ is a subsolution to problem (1).

Next, we consider supersolution to problem (1) in the subcritical and supercritical case, respectively.

Step 2: supersolution for subcritical and critical case: $2<r \leq 2_{s}^{*}$. We look for a supersolution of the form $w(x):=A|x|^{-\beta}$ with $A \geq 0$ and $\beta>0$ as real parameters and verify

$$
\beta<\frac{N-2 s}{2}
$$

Since $r \leq 2_{s}^{*}$, we obtain

$$
(r-1) \beta<\beta+2 s,
$$

$$
\beta r<N
$$

By (49), we deduce that

$$
(-\Delta)^{s} w-\lambda \frac{w}{|x|^{2 s}} \geq w^{r-1}, \quad \text { in } \Omega
$$

for the appropriate choice of $A$.

Let $\eta:=\inf _{\Omega} w>0$. Taking $\bar{u}=C_{1} w$ with $0<C_{1}<1$, which is a suitable constant such that, for $\delta$ small enough, and by (10) we have

$$
\eta^{r-p} \geq \frac{\delta c_{1}}{C_{1}^{1-q}\left(C_{1}-C_{1}^{r}\right)}
$$

where $\delta$ appears in (1).

By (52), we obtain

$$
\begin{cases}\left(-\Delta^{s}\right) \bar{u}-\lambda \frac{\bar{u}}{|x|^{2 s}} \geq \bar{u}^{r-1}+\delta g(\bar{u}), & \text { in } \Omega, \\ \bar{u} \geq 0, & \text { in } \mathbb{R}^{N} \backslash \Omega .\end{cases}
$$

Thus, we have concluded that $C_{1} w$ is a supersoution to (1) for $2<r \leq 2 *$. Moreover, by (48) and (50), we obtain that

$$
\begin{aligned}
& \bar{u} \in L^{r}(\Omega), \\
& \frac{\bar{u}^{2}}{|x|^{2 s}} \in L^{1}(\Omega) .
\end{aligned}
$$

Define $\left\{w_{j}\right\}$ in $L^{1}\left(\mathbb{R}^{N}\right)$ is the weak solution to

$$
\begin{cases}(-\Delta)^{s} \omega_{j+1}=w_{j}^{r-1}+\lambda \frac{w_{j}}{|x|^{2 s}}+\delta g\left(w_{j}\right), & \text { in } \Omega, \\ \omega_{j+1}=0, & \text { in } \mathbb{R}^{N} \backslash \Omega,\end{cases}
$$

for $j \geq 1$ and $w_{0}:=\underline{u}$. We now check that this definition makes sense and $\left\{\bar{w}_{j}\right\}$ are monotone and satisfy

$0 \leq \underline{u} \leq w_{1} \leq w_{2} \leq \cdots \leq w_{j} \leq w_{j+1} \leq \cdots \leq \bar{u}$ a.e. $\Omega$. 
For $\underline{u}$, there is nothing to prove. Suppose the result is true up to order $j$. Then,

$$
\begin{cases}(-\Delta)^{s} w_{j+1}=w_{j}^{r-1}+\lambda \frac{w_{j}}{|x|^{2 s}}+\delta g\left(w_{j}\right), & \\ \leq \bar{u}^{r-1}+\lambda \frac{\bar{u}}{|x|^{2 s}}+\delta g(\bar{u}), & \text { in } \Omega, \\ \omega_{j+1}=0, & \text { in } \mathbb{R}^{N} \backslash \Omega .\end{cases}
$$

So $\left\{w_{j}\right\}$ is well-defined by (54) and Lemma 2. By the induction hypothesis, for $x \in \Omega$,

$$
\begin{aligned}
& (-\Delta)^{s}\left(w_{j+1}-w_{j}\right) \\
& =\left(w_{j}^{r-1}-w_{j-1}^{r-1}\right)+\lambda \frac{\left(w_{j}-w_{j-1}\right)}{|x|^{2 s}}+\delta\left(g\left(w_{j}\right)-g\left(w_{j-1}\right)\right) \\
& \geq 0 \text {, } \\
& \text { and } w_{j+1}-w_{j}=0 \text {, in } \mathbb{R}^{N} \backslash \Omega \text {. Then, by Lemma } 1 \text {, we } \\
& \text { obtain } w_{j+1} \geq w_{j} \text { in } \Omega \text {. } \\
& \text { Similarly, for any } x \in \Omega \text {, } \\
& (-\Delta)^{s}\left(\bar{u}-w_{j}\right) \geq\left(\bar{u}^{r-1}-w_{j}^{r-1}\right)+\lambda \frac{\left(\bar{u}-w_{j}\right)}{|x|^{2 s}}+\delta(g(\bar{u}) \\
& \left.\qquad-g\left(w_{j}\right)\right) \geq 0,
\end{aligned}
$$

and $\bar{u}-w_{j+1} \geq 0$ in $\mathbb{R}^{N} \backslash \Omega$. Then, $w_{j+1} \leq \bar{u}$ a.e. in $\Omega$. We conclude that (56) holds.

We can define $u_{\delta}:=\lim _{j \longrightarrow \infty} w_{j}$ in $L^{1}(\Omega)$. Moreover, by (9), (54), and (56),

$$
\begin{aligned}
\left\|(-\Delta)^{s / 2} w_{j}\right\|_{L^{2}\left(\mathbb{R}^{N}\right)}= & \lambda \int_{\Omega} \frac{w_{j} w_{j-1}}{|x|^{2 s}} \mathrm{~d} x+\int_{\Omega} w_{j} w_{j-1}^{r-1} \mathrm{~d} x \\
& +\delta \int_{\Omega} w_{j} g\left(w_{j-1}\right) \mathrm{d} x \\
\leq & \lambda \int_{\Omega} \frac{\bar{u}^{2}}{|x|^{2 s}} \mathrm{~d} x+\int_{\Omega} \bar{u}^{r} \mathrm{~d} x \\
& +\delta c_{1} \int_{\Omega} \bar{u}^{q} \mathrm{~d} x \leq C .
\end{aligned}
$$

Hence, up to a subsequence, we know that $w_{j} \rightarrow u_{\delta}$ in $X_{0}^{s}(\Omega)$. By monotony, the whole sequence weakly converges. Therefore, we can pass to the limit in (55) and conclude that $u_{\delta} \geq 0$ is a minimal energy solution of (1).

Step 3: supersolution for supercritical case: $2_{s}^{*}<r<r(\lambda, s)$. If $r<r(\lambda, s)$, where $r(\lambda, s)$ is given in
(9). For constant $A \geq 0$, there exists a radial function $v(x):=A|x|^{-2 s /(r-2)}$ such that

$$
(-\Delta)^{s} v-\lambda \frac{v}{|x|^{2 s}}=v^{r-1}, \quad \text { in } \mathbb{R}^{N}
$$

Since $r>2_{s}^{*}>((2 N-2 s) /(N-2 s))$, then

$$
\begin{array}{r}
v \in L_{\mathrm{loc}}^{r-1}\left(\mathbb{R}^{N}\right), \\
\frac{v}{|x|^{2 s}} \in L_{\mathrm{loc}}^{1}\left(\mathbb{R}^{N}\right) .
\end{array}
$$

Taking $\bar{u}=C_{1} v$, where the constant $C_{1}>0$ is given by (54), we obtain

$$
\begin{cases}(-\Delta)^{s} \bar{u}-\lambda \frac{\bar{u}}{|x|^{2 s}} \geq \bar{u}^{r-1}+\delta g(\bar{u}), & \text { in } \Omega, \\ \bar{u}>0, & \text { in } \mathbb{R}^{N} \backslash \Omega .\end{cases}
$$

Moreover by (62), $\bar{u} \geq 0$ satisfies (36). By Lemma 2, define $\left\{w_{j}\right\}$ to be the weak solutions of (55). Moreover, similarly, by the induction hypothesis, we can conclude that

$$
0 \leq \underline{u} \leq w_{1} \leq w_{2} \leq \cdots \leq w_{j} \leq w_{j+1} \leq \cdots \leq \bar{u} \text { a.e. } \Omega .
$$

Note that $(F 2)$ and $(F 4)$ hold, for all $\delta \in\left(\lambda_{1} / M_{0}, \delta_{0}\right]$, define $w_{1}$ as the solution of

$$
\begin{cases}(-\Delta)^{s} w_{1}=\underline{u}^{r-1}+\lambda \frac{\underline{u}}{|x|^{2 s}}+\delta g(\underline{u}), & \text { in } \Omega, \\ w_{1}>0, & \text { in } \Omega, \\ w_{1}=0, & \text { in } \mathbb{R}^{N} \backslash \Omega .\end{cases}
$$

Set

$F(\underline{u})=\underline{u}^{r-1}+\lambda \frac{\underline{u}}{|x|^{2 s}}+\delta g(\underline{u})=\left(t \varphi_{1}\right)^{r-1}+\lambda \frac{t \varphi_{1}}{|x|^{2 s}}+\delta g\left(t \varphi_{1}\right)$.

Then, $w_{1} \in W_{0}^{1, p} \cap L^{\infty}(\Omega)$. By (44), we obtain that

$$
\begin{cases}(-\Delta)^{s} w_{1}=F(\underline{u}) \geq(-\Delta)^{s} \underline{u}, & \text { in } \Omega, \\ w_{1}=0, & \text { in } \mathbb{R}^{N} \backslash \Omega .\end{cases}
$$

We deduce from the comparison principle that $\underline{u} \leq w_{1}$ in $\Omega$. 
On the contrary, by (11), the function $F$ is nondecreasing. Therefore,

$$
\begin{cases}(-\Delta)^{s} \bar{u} \geq F(\bar{u}) \geq F(\underline{u})=(-\Delta)^{s} w_{1}, & \text { in } \Omega, \\ \bar{u} \geq w_{1}, & \text { in } \mathbb{R}^{N} \backslash \Omega .\end{cases}
$$

By the comparison principle we deduce that $w_{1} \leq \bar{u}$ in $\Omega$. In particular, for all $x \in \Omega,\left\{w_{j}\right\}$ is a nondecreasing sequence which is bounded. Therefore, $\left\{w_{j}\right\}$ monotone converges in $L^{1}\left(\mathbb{R}^{N}\right)$ to a weak nonnegative solution $u_{\delta}$ to (1) for $2_{s}^{*}<r<r(\lambda, s)$.

Therefore, for $\delta$ small enough, we have built a minimal solution in both subcritical and supercritical case. Let

$$
M=\sup \{\delta>0: \text { problem (1) has a solution }\},
$$

that is, we show that $M>0$.

Step 4: $M<\infty$, for $2<r<r(\lambda, s)$. We consider the following eigenvalue problem with the Hardy potential:

$$
\begin{cases}(-\Delta)^{s} \psi_{1}-\lambda \frac{\psi_{1}}{|x|^{2 s}}=\lambda_{1} \psi_{1}, & \text { in } \Omega, \\ \psi_{1}=0, & \text { in } \mathbb{R}^{N} \backslash \Omega .\end{cases}
$$

Since $0<\lambda<\Lambda_{N, s}$, problem (70) is well defined. Taking $\psi_{1}$ as a test function in problem (1), we obtain that

$$
\begin{aligned}
& \frac{C(N, s)}{2} \int_{Q} \frac{\left(\psi_{1}(x)-\psi_{1}(y)\right)(u(x)-u(y))}{|x-y|^{N+2 s}} \mathrm{~d} x \mathrm{~d} y-\lambda \int_{\Omega} \frac{\psi_{1} u}{|x|^{2 s}} \mathrm{~d} x \\
& \quad=\int_{\Omega} u^{r-1} \psi_{1} \mathrm{~d} x+\delta \int_{\Omega} g(u) \psi_{1} \mathrm{~d} x .
\end{aligned}
$$

Since $\psi_{1}$ is a solution to (70), it follows that

$$
\int_{\Omega}\left(u^{r-1}+\delta g(u)\right) \psi_{1} \mathrm{~d} x=\lambda_{1} \int_{\Omega} u \psi_{1} \mathrm{~d} x .
$$

If $2_{s}^{*}<r<r(\lambda, s)$. Taking $\varphi_{1}$ as a test function in (1), where $\varphi_{1} \geq 0$ is solution to problem (44), we have

$$
\begin{aligned}
\int_{\Omega} u(-\Delta)^{s} \varphi_{1} \mathrm{~d} x & =\int_{\Omega}\left(\lambda \frac{u}{|x|^{2 s}}+u^{r-1}+\delta g(u)\right) \varphi_{1} \mathrm{~d} x \\
& \geq \int_{\Omega}\left(u^{r-1}+\delta g(u)\right) \varphi_{1} \mathrm{~d} x .
\end{aligned}
$$

Moreover, $\varphi_{1}$ is also a classical solution (see Remark 2.1 in [21]). From (72), we immediately deduce that

$$
\lambda_{1} \int_{\Omega} u \varphi_{1} \mathrm{~d} x \geq \int_{\Omega}\left(u^{r-1}+\delta g(u)\right) \varphi_{1} \mathrm{~d} x .
$$

Since there exist structural positive constants $b_{0}$ and $b_{1}$ such that $|t|^{r-2} t+\delta g(t)>b_{0} \delta^{b_{1}} t$, for any $t>0$. From (70) and (73), we obtain that $b_{0} \delta^{b_{1}}<\lambda_{1}$. This implies that $M<\infty$ for $r<r(\lambda, s)$.

We complete the Proof of Theorem 1.

\section{Nonexistence Result}

In this section, we consider the nonexistence of solution to problem (1) in $H_{0}^{s}$.

Proof of Theorem 2. Suppose that problem (1) has a solution $u \in H_{0}^{s}$ under the conditions of Theorem 2 . Then, there exists a constant $\delta>0$ such that $\delta \varphi_{1} \leq u$ in $\Omega$, where $\varphi_{1}$ is the first eigenfunction of $(-\Delta)^{s}$, that is, $\varphi_{1}$ satisfies (44).

Let $\mu \in\left(\lambda_{1}, \lambda_{1}+\varepsilon\right)$, where $\varepsilon>0$ is a small constant. Denote $\psi=\delta \varphi_{1}$. Then, we have

$$
\begin{cases}(-\Delta)^{s} \psi=\lambda_{1} \psi \leq \mu \psi<\left(\lambda_{1}+\varepsilon\right) u, & \text { in } \Omega, \\ \psi=0, & \text { in } \mathbb{R}^{N} \backslash \Omega .\end{cases}
$$

Furthermore, for any $\tau>1, \delta<\lambda_{1} / M_{1}(\tau)$; then, for $\varepsilon$ small enough, we deduce that

$$
\begin{cases}\left(\lambda_{1}+\varepsilon\right) u \leq u^{r-1}+\delta g(u)+\lambda \frac{u}{|x|^{2 s}} \equiv(-\Delta)^{s} u, & \text { in } \Omega, \\ u=0, & \text { in } \mathbb{R}^{N} \backslash \Omega .\end{cases}
$$

Thus, according to (75) and (76), we have

$$
\begin{cases}(-\Delta)^{s} \psi \leq \mu \psi, & \text { in } \Omega, \\ (-\Delta)^{s} u \geq \mu u, & \text { in } \Omega, \\ u \geq \psi, & \text { in } \Omega, \\ u=\psi=0, & \text { in } \mathbb{R}^{N} \backslash \Omega .\end{cases}
$$

Hence, it is possible to construct the subsolution and supersolution to the problem:

$$
\begin{cases}(-\Delta)^{s} u=\mu u, & \text { in } \Omega, \\ u=0, & \text { in } \mathbb{R}^{N} \backslash \Omega,\end{cases}
$$

with $\mu \in\left(\lambda_{1}, \lambda_{1}+\varepsilon\right)$. However, this is impossible.

\section{Data Availability}

Data sharing not applicable to this article as no datasets were generated or analysed during the current study.

\section{Conflicts of Interest}

The authors declare that they have no conflicts of interest. 


\section{Acknowledgments}

This research was partially supported by the National Natural Science Foundation of China (no. 11761059), Program for Yong Talent of State Ethnic Affairs Commission of China (no. XBMU-2019-AB-34), Fundamental Research Funds for the Central Universities (no. 31920200036), and First-Rate Discipline of Northwest Minzu University.

\section{References}

[1] S. Huang and Q. Tian, "Marcinkiewicz estimates for solution to fractional elliptic laplacian equation," Computers \& Mathematics with Applications, vol. 78, no. 5, pp. 1732-1738, 2019.

[2] S. Huang and Q. Tian, "Harnack-type inequality for fractional elliptic equations with critical exponent," Mathematical Methods in the Applied Sciences, vol. 43, no. 8, pp. 5380-5397, 2020.

[3] M. Ri, S. Huang, and C. Huang, "Non-existence of solutions to some degenerate coercivity elliptic equations involving measures data," Electronic Research Archive, vol. 28, no. 1, pp. 165-182, 2020.

[4] L. Silvestre, "Regularity of the obstacle problem for a fractional power of the laplace operator," Communications on Pure and Applied Mathematics, vol. 60, no. 1, pp. 67-112, 2007.

[5] Q. Tian and Y. Xu, "Effect of the domain geometry on the solutions to fractional brezis-nirenberg problem," Journal of Function Spaces, vol. 2019, Article ID 1093804, 4 pages, 2019.

[6] Y. Ye, H. Liu, Y. Wei, M. Ma, and K. Zhang, "Dynamic study of a predator-prey model with weak Allee effect and delay," Advances in Mathematical Physics, vol. 2019, Article ID 7296461, 15 pages, 2019.

[7] B. Abdellaoui, M. Medina, I. Peral, and A. Primo, "The effect of the hardy potential in some Calderón-Zygmund properties for the fractional laplacian," Journal of Differential Equations, vol. 260, no. 11, pp. 8160-8206, 2016.

[8] Y. Mi, S. Huang, and C. Huang, "Combined effects of the hardy potential and lower order terms in fractional laplacian equations," Boundary Value Problems, vol. 2018, no. 1, p. 61, 2018.

[9] B. Barrios, M. Medina, and I. Peral, "Some remarks on the solvability of non-local elliptic problems with the hardy potential," Communications in Contemporary Mathematics, vol. 16, no. 4, Article ID 1350046, 2014.

[10] X. Shang, J. Zhang, and R. Yin, "Existence of positive solutions to fractional elliptic problems with hardy potential and critical growth," Mathematical Methods in the Applied Sciences, vol. 42, no. 1, pp. 115-136, 2019.

[11] K. Bogdan, T. Grzywny, T. Jakubowski, and D. Pilarczyk, "Fractional laplacian with hardy potential," Communications in Partial Differential Equations, vol. 44, no. 1, pp. 20-50, 2019.

[12] S. Dipierro, L. Montoro, I. Peral, and B. Sciunzi, "Qualitative properties of positive solutions to nonlocal critical problems involving the Hardy-Leray potential," Calculus of Variations and Partial Differential Equations, vol. 55, p. 99, 2016.

[13] N. Ghoussoub, F. Robert, S. Shakerian, and M. Zhao, "Mass and asymptotics associated to fractional Hardy-Schrödinger operators in critical regimes," Communications in Partial Differential Equations, vol. 43, no. 6, pp. 859-892, 2018.

[14] S. Rastegarzadeh and N. Nyamoradi, "Existence of positive solutions for hardy nonlocal fractional elliptic equations involving critical nonlinearities," Topological Methods in Nonlinear Analysis, vol. 53, pp. 731-746, 2019.

[15] L. Boccardo, M. Escobedo, and I. Peral, "A Dirichlet problem involving critical exponents," Nonlinear Analysis: Theory, Methods \& Applications, vol. 24, no. 11, pp. 1639-1648, 1993.

[16] L. Caffarelli and L. Silvestre, "An extension problem related to the fractional laplacian," Communications in Partial Differential Equations, vol. 32, no. 8, pp. 1245-1260, 2007.

[17] J. Dávila, L. Dupaigne, L. Dupaigne, and M. Montenegro, "The extremal solution of a boundary reaction problem," Communications on Pure \& Applied Analysis, vol. 7, no. 4, pp. 795-817, 2008.

[18] R. L. Frank, E. H. Lieb, and R. Seiringer, "Hardy-Lieb-thirring inequalities for fractional schrodinger operators," Journal of the American Mathematical Society, vol. 21, no. 4, pp. 925950, 2008

[19] R. Servadei and E. Valdinoci, "Mountain pass solutions for non-local elliptic operators," Journal of Mathematical Analysis and Applications, vol. 389, no. 2, pp. 887-898, 2012.

[20] E. Di Nezza, G. Palatucci, and E. Valdinoci, "Hitchhiker's guide to the fractional sobolev spaces," Bulletin des Sciences Mathématiques, vol. 136, no. 5, pp. 521-573, 2012.

[21] X. Ros-Oton and J. Serra, "The dirichlet problem for the fractional laplacian: regularity up to the boundary," Journal de Mathématiques Pures et Appliquées, vol. 101, no. 3, pp. 275302, 2014. 